InVisible Culture Journal

"I'm Controlling and

Composing": The Role of

Metacognition in The

Incredible Machine

Marc Ouellette ${ }^{1}$

10ld Dominion University

Published on: Apr 18, 2019

License: Creative Commons Attribution 4.0 International License (CC-BY 4.0). 


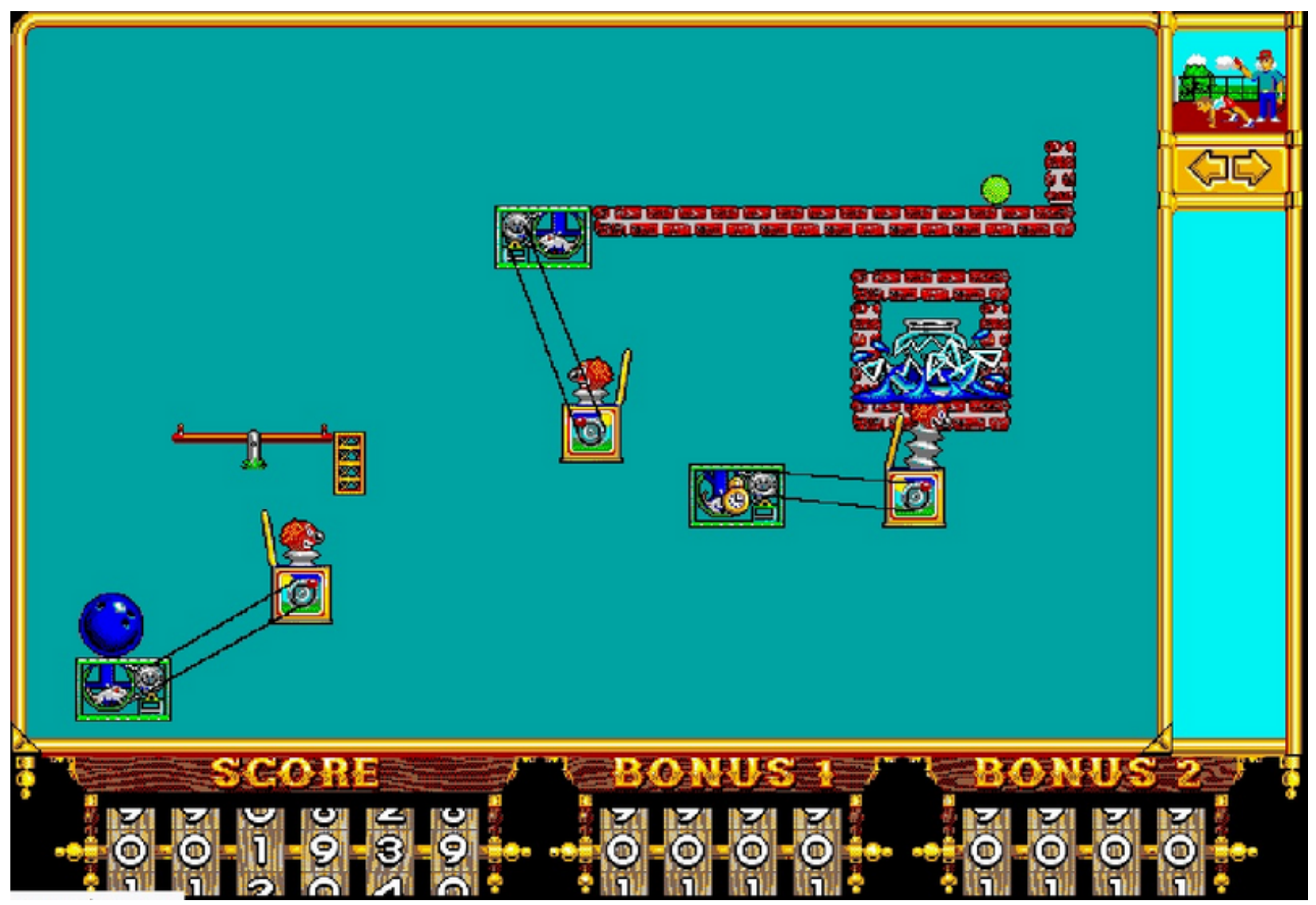

\section{Up, Up, and Away: Introduction}

The mouse sets the bowling ball in motion, which falls and squeezes the bellows, which sends out a puff of air, which sends the balloon into the gears that are connect by a belt to another mouse's exercise wheel. $\underline{1}$ The balloon pops. Having learned how this routine functions, I then move my mouse to connect the rest of the on-screen mice so that the pulleys of all of the caged mice spin with their wheels to finish the puzzle in time allowing me to move to the next level. Eventually, I will be able to make my own versions of Rube Goldberg machines turned into puzzles based on what I have seen and learned in playing through the eighty challenges provided for Mort the mouse, Bob the fish, and me. Although it is more than twenty-five years old, by teaching about games, learning through games, and learning itself, The Incredible Machine (Dynamix, 1992) continues to defy several key deterministic viewpoints about video games. Said another way, The Incredible Machine (TIM) anticipates Douglas Hacker's defining study of metacognition-i.e., "knowledge of one's own knowledge processes"in games and simulations. $\underline{2}$ As a game about learning, TIM resists the prevailing scholarly notion that pleasurable games must follow the cultural imperative for 
accumulation, competition, and/or conquest and that pleasurable games are not suitable for teaching. $\underline{3}$ Indeed, Hacker's later work emphasizes so-called "serious games," which are explicitly and didactically aimed at learning, a process that has "met with mixed results." $\underline{4}$ Thus, my paper will examine the metacognition that occurs in and through the very unserious playing TIM.

While the solutions to the puzzles help make TIM a pleasurable game, the process also provides several important commentaries on games themselves, particularly how games teach learning strategies, or meta-cognition, for digital scenarios. Three related factors in the game's design, then, provide the means for the metacognition. First, completing any puzzle in TIM requires the reproduction, replication, and repetitions of the ubiquitous Programmer's Algorithm, namely to define the problem and then plan, code, debug and share a solution. $\underline{5}$ Following from this, playing through the puzzles affords and relies on the freedom of the free play mode by virtue of this approach. Starting with the hints, players are encouraged to experiment and to learn the game's lessons as they learn its elements. This second feature, which Conway calls "ludicity," refers to "the degree to which digital games allow play." $\underline{6}$ The iterative process through which the ludicity occurs also combine with the third element, "metagaming," which Jensen argues is a "relatively unknown concept" in game studies. $\underline{7}$ Metagame features are generally understood to entail rewards that are external to the game as well as player-defined goals and achievements. While this is the very contingency of TIM's creator mode, the elements, both about games and programming, along with rewards for following it, that the game makes its most significant statement. As Nohr observes, any metagame or metagame feature is always already a comment on games, in general. $\underline{8}$ This is important because it becomes clear that TIM bridges the gap Ruggill and McAllister enumerate as marking game studies, one between "what games can be" and "what games are." $\underline{9}$ Ultimately, TIM provides eighty levels that map the ways games can and do teach without being serious and do so in games that are pleasurable without being competitive, both of which scholars such as Vorderer, Hartmann, Klimmt, and Oliver et al insist are necessary for successful games that teach. $\frac{10}{}$

However, in its deployment of the Programmer's Algorithm, metagame features and the ludicity that facilitates the two, it becomes clear that TIM can teach literaciesnamely programming, game design, and algorithms-beyond the play of games. In fact, this solves the major problem that occasions Hacker's rethink of the failures of serious games. He acknowledges that "high engagement with video games enhances a 
person's ability to engage in video games." $\underline{11}$ Moreover, Hacker continues his consideration of the failures of serious games by citing three other works that find similarly, that the "research on the educational value of edutainment is greatly lacking." 12 In other words, the focus has been what games are or on what games can be. The question them becomes whether there is any benefit to games, or better yet, can a benefit be produced or at least induced in and through video game play. Facilitating metacognition is the essential part, then, of any game that teaches. Simply put, TIM teaches strategies for learning because of its play, not its seriousness, requires it. This is actually the very contingency of Hacker's further elaboration of meta-cognition, for TIM also establishes "an awareness of oneself as a learner" through its game as lesson routines. $\underline{13}$ The multiple and simultaneous opportunities to learn make TIM function as a metagame for and about meta-cognition, and vice versa.

The ultimate reward, the creator mode, serves as an in-game and out-of-game reward since players must complete the game's puzzles before they can contemplate making their own. $\frac{14}{14}$ However, each new element and new level is itself a reward if only because of the deliberate humor involved in them. Through its reward structure, TIM anticipates and confirms Espen Aarseth's assertion that the "gameworld is its own reward." 15 Yet, this is another way of describing ludicity, for the elements of game design are linked ineluctably. Indeed, playing the game, replaying the game (particularly via online emulations), and especially watching the many Let's Play videos on YouTube and elsewhere suggests that Aarseth may have under-estimated the multiple and simultaneous means through which the game rewards players with the game itself. While he cites the tendency for games to involve a progression through game as the typical means through which a game offers, but also limits its rewards, the process applies only to one cognitive and affective response to the regular play mode. Therefore, in the most important parts of its reward system, TIM teaches learners strategies for learning - that is, how to learn - through an engagement with and an enumeration of methods for manipulating systems of logic through the ongoing, active deployment of practices and methods of that system. $\underline{16}$ Thus, the ludicity, the metagame aspects, and the algorithmic nature of the game mechanic combine to produce a game that is neither serious nor competitive but is fun and educational. It should not, then, just be the fact that it includes a design mode that underscores TIM as providing an exemplar, if not a design heuristic, for games that resist these supposed truisms. Indeed, it was playing and writing about Portal and Portal 2 that reminded me of the ways TIM anticipates the features of the later games, which have achieved almost cult-like status, and Oregon Trail, which has. $\frac{17}{}$ 


\section{No Strings Attached: Playing with the Gameworld}

As well, I was reminded of the ways these games illustrate the very contingency that led Perron to distinguish between "players," those who accept a game's limits, and "gamers," those who manipulate and play with those limits. $\underline{18}$ This becomes important because in so doing TIM offers a commentary on games, and by extension, those who play games and, more importantly, it provides a pedagogical means of understanding both through an interface based on the Programmer's Algorithm. Stated simply TIM exists in and through the stepwise process from defining the problem through to documenting a solution. Here, it is important to note the switch from the definite to the indefinite article since there may well be more than one valid solution to any given puzzle. This fact only reinforces the need to plan, to code and to debug that solution. Moreover, as games and game industry have galvanized around competition and accumulation as the rationale and outcome for games, TIM then becomes a space, a means and an exemplar for questioning the determinism that these are necessary for a successful-and pleasurable-game. Moreover, the combination of ludicity, metagame features, and experimentation resist the rigidity of serious games, which demand a didactic "emulation of the expert model," not to mention the insistence that only serious games can teach. $\underline{19}$ As Michel de Certeau writes, this kind of resistance "redistributes its space; it creates at least a certain play in that order, a space for maneuvers of unequal forces and for utopian points of reference." $\underline{20}$ This refers to the hit-and-run tactics of (micro)resistance based on the manipulation of the tendencies of the dominant system against itself. It becomes apparent through playing any mode of $T I M$, that the gameworld occasions, encourages and teaches such manipulations so that these become the rationale and the outcome for playing the game. Playing TIM, then, calls into question the determinism of narrative, resolution, scoring systems and, indeed, game criticism-both popular and scholarly-based on those assumptions. TIM resists the notion that games for learning pro-social behaviors must be explicitly so and/or didactic, as well as the opposing notion, that pleasurable games teach only bad habits if they teach anything at all.

In place of a narrative, it offers a mean of evaluating the products of that system, for as Nohr explains of Portal, the appropriation of the means of production offers a powerful form of dissidence in the metagame. $\frac{21}{T I M}$ includes as its very kernel, and as its central intra-diegetic reward, a means of developing, enjoying, and sharing alternatives to that system and learning how to do it. Thus, a consideration of the ways TIM facilitates metacognition must offer a ludic framework that will in turn examine how the game comments on and contributes to an understanding of games and will 
consider the cognitive and affective means through which this is achieved. More than a metagame about meta-cognition, the game scaffolds the learning through the addition and elimination of supports for learning, particularly strategies for problem solving. This becomes especially important given it is actually the limits and the requirements of the puzzle mode that provide the means and the methods for the experimentation of the free play mode. Outlining the ways in which TIM serves as a (prototype) metagame affords not only a consideration of TIM and the gamification of assessment and surveillance, but also an opportunity to reconsider gamification, particularly in term of its manipulation. As Cole and Jensen agree, not only are metagames under-examined, players find in-built achievements are less rewarding

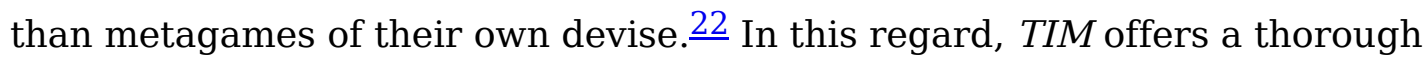
evaluation of what it means to be playful and anticipates what Fest calls the "metaproceduralism" of The Stanley Parable. $\underline{23}$ TIM puts paid to questions of whether or not games teach while also transcending questions of whether games need to be covert in offering their pedagogies. Playing TIM means learning how to learn in and through strategies of learning. As a corollary, then, a study of TIM almost necessarily questions the determinism of pleasure as a necessary component of gameplay.

As much as it might be presumed that the free play mode of TIM exists without the rules, encumbrances and limitations of the kernel game, it actually exists in and through the constraints. Each level of TIM opens with a hint, which shows how a particular game element-scissors, motors, generators, etc.-can be used or combined. As well, the player is given a set of items which may be used together or individually to create a solution. Motors and generators require belts to connect them to devices or to be connected. Some elements, like treadmills, work with belts and motors, and can be turned to spin in a different direction. Usually, the player is given more parts than are necessary to complete a level and there are usually more than one solution. Paradoxically, though, the various constraints-of parts, mechanisms, object, and space -become key components of the central reward of the game, for solving the puzzles of the regular mode provides hints and examples of the functions and the operations of the game elements. More than just the means for solving puzzles, the game elements and their roles provide their own learning mechanisms. As early as the third level, "Bellows and Balloons," which requires pushing three balloons with bellows so that they can be burst with either scissors or spinning gears, can be solved by first having a dry run of the given mechanisms or setting up trial mechanisms, as well as breaking the solution into pieces. By the fourth level, "Flip, Flip, Flip," which requires using a series of see-saws in a combination to bounce balls over a set of barriers, the player 
can begin breaking the problem into iterative pieces. The level starts with two seesaws already in place, with bowling balls on the lower end of the see-saw. A dry run reveals immediately the way the machines function individually and together. A Programmers might call these subroutines; teachers should recognize that this is a form of "chunking." In other words, without being didactic, the game begins and proceeds by offering insights into strategies for learning. In fact, the two skills just listed become necessary in solving the sixth puzzle, "Bouncing Over to Mort," which involves placing a series of trampolines in a step-wise fashion in order to amplify a ball's bounces so that it drops into an elevated basket. Without advertising it as such, the game has just taught an iterative process and debugging. However, it is well worth noting that the player also has the freedom-that is, the ludicity-to make mistakes and learn from them. This affords greater access to the game and the skill learned in the fourth puzzle, iterative processes, has to be transferred from one device (see-saws) to another (trampolines) in order to solve the puzzle. The ability to transfer a learned skill is itself a metagame feature for it offers a commentary on the very nature of the play.

As a further example, it is only after playing a level such as the twelfth puzzle, "Generators and Motors" that a player can tell not only the difference between a motor and a generator in the game, but also the operations and constraints of those elements. The former needs to be connected to a power outlet while the latter becomes a replacement for the outlets. Similarly, the very first level reveals that mouse cages must be bumped in order for "Mort," the mouse, to start running. He can then power belts, but also generators and other things. In this way, at least at a metatextual level, TIM incorporates the features of what Ruggill, McAllister and Menchaca call, "the gamework." $\underline{24}$ In considering the potential for games to become sites of work, Ruggill, et al., highlight the extent to which those playing games, "actually help [to] create the narrative, the metric, and ideological structures that determine the artifactual experience. In so doing, gamers also reproduce or consent to ideologies embedded within games themselves." 25 This insight is significant for an understanding of TIM teaching learners how to learn, by teaching strategies that might be useful for any game in at least three ways.

First, it offers a very clear metagame reminder that as Ouellette and Ouellette argue, play always already involves compromise. $\underline{26}$ The need is as much one of balancing available items as it is one of survival. For its part, TIM involves several resources and with them a series of compromises, ranging from the time bonuses for speedy 
completion to the number of pieces used to solve a puzzle. In detailing the metacognitive potential of what he calls "serious games," Hacker argues that these involve "a person's ability (a) to allocate his or her resources to the current task, (b) to determine and direct the steps to complete the task, (c) to set the intensity or (d) the speed of the work task." $\underline{27}$ Indeed, the tenth level of The Incredible Machine includes a red herring to mislead players. This last element also reminds players that one of the key resources can be labour. The rationing and/or careful apportioning of labour occurs most notably if players cooperate in creating or in solving puzzles but also through the very act of agreeing to the game's governing constraints. However, it is well worth noting that reading TIM in this way offers insights applicable to other games. A common example is trading off collecting every available item for the sake of speed. Perhaps the most famous instance of a playful compromise is the dilemma of Missile Command, which requires that players decide between protecting cities or protecting missile bases. Alas, the cities must be sacrificed in order to win. Beyond being a moral decision or a decision based on expedience, this also reveals the necessity for resource management, planning, and strategies for both. Not only is this a key component of metacognition-awareness of one's self as a learner-it also offers insights into the ways games can teach, and indeed are teaching, without being "serious."

While the game does not have an explicit multi-player component, there is nothing at all preventing players from joining together, plotting, comparing and testing solutions. The last two of these constitute very common metagame operations and these provide the most clear instance not only of commenting on the game and the play of the game. In turn, considering these metagame aspects offers insights into the development of a collaborative form of learning and the need to develop strategies for it. For example, as soon as the fourth puzzle, those playing need to create subroutines, iterations, and dummy routines. The game's in-built pedagogical routines then operate explicitly and implicitly depending on the level of player adaptation and cooperation. Second, TIM does not so much have a narrative as it actively seeks the creation of one and simultaneously comments on (the making of) games and puzzles and learning how to solve them. On its face, the game simply is an insistent one, given the time counter and the requirement to solve the puzzles in order to proceed to subsequent levels and to receive the creative mode. However, the game is also one of building, exploring and collecting and it is these aspects that provide players with a high degree of ludicity in order to create their own narrative through the need to understand how to construct a level, how to combine and place the elements, as well as how to implement what has 
been gained (and learned). In terms of the effectiveness of meta-cognitive routines in games, Mayer notes that one of the key challenges when employing games as a means of facilitating metacognition that "learners are unschooled about tools for studying effectively." 28 The tools might not always seem obvious since games are not always didactic. In addition to the multiple solutions and the presence of red herrings, TIM introduces counter-intuitive and/or multi-dimensional thinking in level eleven, "Like a Hurricane," which has a light switch that is activated by a ball moving upward, not a falling one, as in previous levels, in order to activate the last of three fans required to complete the puzzle. The player must figure this out only after connecting all of the various elements Using the balls in multiple directions and modes becomes useful in succeeding levels. The very next puzzle, "Bang, Bang, Bang," for instance, can be solved as a series of continuing movements to fire cap guns or each one can be treated as a discrete entity. Not only does the puzzle solution invite multiple solutions, it begs collaboration, as well. After all, sharing one's own puzzles is the ultimate reward of the game. Beyond the challenges noted above, Mayer cautions that "open environments place greater demands on the learner" trying to develop strategies in and through game-based interactions. $\frac{29}{}$ An essential part of any player's personal narrative comprises means and methods of learning as well as the supports for them. Thus, it is no surprise that in each of the instances listed above, TIM includes the player in a very clear process of instructional scaffolding. That is to say, the games adds and removes supports depending on what the player can (by virtue of having made it this far) and cannot do with (or without) assistance. The game offers hints but also adds and removes parts and preset mechanisms. The last then becomes a series of demonstrations (i.e., exemplars) that tap recall and recognition, as well as application and adaptation so that the game elements combine to facilitate a collaborative form of metacognition. Indeed, even the addition of hints makes the game always already a collaborative one.

As a puzzle game, TIM, can be included quite easily among the games that correspond with Aarseth's insights on the necessary relationship between narrative and play. However, TIM also suggests that one might place equal emphasis on each part of the narrative-rules, rewards and recreation-that produce the cognitive and affective dimensions of games. On the surface, Aarseth's explanation of the intersection of narrative and play favors play since he argues that stories limit video games in "unrealistic ways. What makes such games playable at all, and indeed attractive, is the sequence of shifting, exotic, often fascinating settings (levels), where you explore the topography and master the virtual environment. The gameworld is its own reward." $\underline{30}$ 
As much as it seems that Aarseth's position values the reward structure of games, it really attributes a game's success to its ludicity and structure as a game rather than the ability to play with its reward. This is another instance of the earlier cited gap between thinking of what games can be or what games are. One of the ways TIM bridges this gap is to produce the game world as rationale and outcome through the advancement from level to level, the addition of pieces and routines, access to the creative mode, and especially the series of learning-and indeed, metacognitveprocesses that facilitate and underlie each aspect of the progression. While it is easy to agree that form is content, recognizing the relationship between structure and subject does not fully account for the multiple and simultaneous ways that a gameworld can become its own reward through the interplay of the formal elements and the work of play. This is important because the cognitive and affective responses contingently centre on the interplay of the user defined achievement, such as solving a puzzle cooperatively, differently or competitively, as well as the external reward structure, most obviously presented in the creative mode. By literally playing with the idea that work is not pleasurable, TIM again defies the prevailing logic about pleasurable games and about games that teach needing to be serious. Since it is a metagame, it also offers insights into how similar games can be created.

In terms of the design of TIM's ludic structure, the production of cognitive and affective responses to the game's metacognitive properties occurs in two distinct but related ways. First the game highlights Perron's previously cited enumeration of "player" and "gamer." Of the former, Perron explains, "players know they are playing and being played." $\underline{31}$ To some extent, the duality of playing and being played is always true for any attempt to play TIM, in either mode. The range of possibilities includes extraneous bits, extra manipulable pieces, and surprises like the jack-in-a-box switching from Mort to Bob. However, submitting to the rules and constraints of the gameworld facilitates and enhances the opportunity to become a gamer, or one for whom, it is not "a question of playing the game but of playing freely with the game." $\underline{32}$ More important, though, is the means through which TIM facilitates such negotiations of the game's very structure, particularly by virtue of its deployment and or implementations of the oft-cited Programmer's Algorithm. $\underline{33}$ Overtly or not, learning from the algorithm and its process becomes one of the key rewards of the game and in several different ways. At its simplest, the need to test and to debug becomes another layer of the reward, as do the elements learned through testing and debugging. The latter provides a key pillar of scaffolding, namely the anticipation of the sorts of errors that learners might make as they attempt a new task. In Hacker's subsequent 
elaboration, he explains that metacognitive operations proceed from "a person's ability (a) to identify the task on which one is currently working, (b) to check on current progress of that work, (c) to evaluate that progress, and (d) to predict whether the expected outcome will be attained." $\underline{34}$ As with the earlier-cited steps in identifying resources, these steps map onto the Programmer's Algorithm and vice versa and the processes contribute to the scaffolding of the game. For example in "Puzzle 6 Tutorial: Bouncing over to Mort," the player must place the trampolines and test their placement repeatedly to get the ball to bounce to the correct destination. Moving one trampoline necessitates moving all remaining trampolines in the sequence. These moves then require a determination of where the ball will hit each trampoline each time, as well as accounting for the ball's velocity and angle. Finally, the opportunity to experiment with the structure of the game makes the free play mode a kind of commentary on the learned game by virtue of the pedagogical impact of the process and the potential for comparative evaluation. Thus the gameworld is its reward, but not simply because of the variety of settings and locales. Rather, it develops practices and attitudes, all of which induce and produce moments of learning, particularly strategies of learning, that reward the player within TIM and in future games, as well.

\section{Follow the Bouncing Ball: The Programmer's Algorithm and Play}

Ultimately, TIM teaches gamers about games, how to play them, and how to learn about learning, especially through the steps of the Programmer's Algorithm.

Recognizing that the gameworld is its own reward leads to several important insights about the commentary TIM produces. The puzzles not only teach players how the elements work, they teach you how to play the succeeding levels of the game and, more importantly, how to make the most of the experimentation of the free play mode. For Mayer, a foundational aspect of metacognition derives from an "awareness and control of one's own cognitive processing." $\underline{35}$ The necessity of defining a problem thoroughly, the first requirement of the method, can require (a) deliberate failure in order to learn the extent of boundaries, of motions, of combinations, and of installed pieces. Learning the boundaries of the gameworld and the fail conditions, as opposed to win conditions, provides the awareness and the basis for control. For instance, the need to press the button to "run" the test, in a kind of dry run before even considering a solution becomes clear almost immediately. The first instance occurs in the third level, which requires the player to use fans, gears, and scissors to burst a handful of balloons. Before starting a solution, it becomes necessary to see which balloons will burst without intervention, which ones move upon activation, and which ones need a push. At the very least, this move allows the player to see what all the items on the 
screen do without any intervention. It also provides examples of ways to use particular elements for future creations, as well as revealing alternative means of activating devices. Part of coding a solution requires knowing the functions of the devices and their effects. One of the particularly challenging pieces is the see-saw. They can be used to fling objects, in the expected manner. However, a rope or string can be tied to an end so that raising or lowering that end causes the string to pull the cord on a light switch or to lift a bucket. Even so, the movement is not always sufficiently powerful, so a pulley must be added or a substitution involving a different mechanism must be made. Not only does this highlight debugging and planning, it also invokes the importance of documentation and distribution, even if the player's memory and catalogue of solutions are the basis of the record. It was also very beneficial to discover that almost any round object could be acted upon by gravity and therefore could be used to activate switches, flashlights, and similar items.

In solving "Puzzle 48: Exercise Kelly the Monkey," making the monkey exercise is not only the goal, it involves learning that a string needs to be pulled to activate the Venetian blinds that hide the banana that provides the enticement for riding the (stationary) bicycle. More than this, the combination becomes useful later, as does learning how to connect a pulley belt to the bicycle. Moreover, there is no penalty for making this move. Indeed, since the timer and the points start counting down right away, there is little benefit to not enacting a dry run. In fact, the timer that removes points stops while the game is being run to test it. Thus, in playing the free, creative mode, making use of the "dry run" in creating the puzzle, helps to anticipate and to plan for its inclusion in solving the puzzle. Thus, anticipating error is a condition of all aspects of TIM. In this way, it scaffolds the learning as well as the mechanism for learning. It becomes quite clear, then, that not only are there benefits to creating and to solving the puzzles through testing, the game calls into question the determinism of "victory" as the defining criteria for success in games through its redeployment of failure as a pedagogical moment, one that demonstrates that success in learning has multiple and simultaneous outcomes, that the means is as important as the end, and that there are different paths to achieve a solution. Indeed, in scoring the levels, the bonus countdown pauses during testing. Rather than a linear shooter, the entirety of the game becomes a series of instructional levels, teaching players how to use the objects, how to test and re-test until the desired effect is achieved, and how to do clever (or silly) things with a bucket, a mouse and some dynamite. This also means there really is no failure because while you may not figure out how to use everything in the free mode, it can still be accessed at any time. What becomes significant, then, is 
that players must learn to anticipate errors - theirs and the ones made by others who receive the new levels-in order to create a playable level.

Therefore, after playing both modes of the game several times, subsequent play echoes and/or maps onto the form-consciously or not, intentionally or not-of the five steps of the Programmer's Algorithm: define the problem, plan a solution, code the solution, test and debug the solution, and document that solution. What becomes clear is that the steps of the algorithm map onto the process of metacognition and vice versa. As Hacker originally defines it, metacognition includes knowledge of the learning processes along with the contingent "ability to consciously and deliberately monitor and regulate one's knowledge, processes, and cognitive and affective states." $\underline{36}$ As is typical of a study of metacognition and games, however, the focus remains on serious games. In the example of Puzzle 48, the game's definition of the given problem is not sufficient. Indeed, it is no more sufficient than any of Portal's test areas or Minecraft's cake recipe, to cite just two equally playful later games that can be similarly considered. In all cases, several other processes and tasks must be performed separately, in advance and/or concurrently-that is, synchronous, asynchronous and hybrid, as one might say of distance learning -in order to even begin the actual task. Thus, defining a problem thoroughly and accurately, including all subroutines and parallel processes, becomes a basic requirement, one that is scaffolded throughout. Debugging and dissemination each offer a means of monitoring, with the former, especially providing the basis for regulation. Testing and sharing become opportunities to determine the usability or playability of a given puzzle and/or a given solution. Playing through the puzzles provided with the game facilitates greater freedom in the free play mode by virtue of the step-wise approach and the lessons it teaches. Indeed, Hacker continues to stress that games "need to provide a sense of agency within a game, and yet that very agency is compromised by the need to provide players with explicit guidance for learning. Agency is built into game designs by creating open environments." $\underline{37}$ Yet, this need not be an exclusive case. Again, it is well worth mentioning that Hacker also continues to focus on games that are solely built to teach. Metagame aspects offer an immediate index of ludicity and TIM offers all of the features Hacker lists prior to the opening of the free mode. The first clue that the Programmer's Algorithm is an incredibly useful approach comes at the beginning of each level with the given task and the basic hints. This moment in the game also invites the dry run to begin the planning process by combining the behavior of the elements with the tools in the menu along with the objects and empty space on the screen. 
Conversely, creating a puzzle requires the same operations, if not in the same order in an iterative sequence since coding the solution only works after testing and debugging the solution. The solution is formalized and documented once the replay button appears. Thus, the "win condition" lies in understanding how to manipulate the elements, in either mode. Thinking of the game world really as its own reward becomes an iterative process because you can go to the next level, get a password to skip levels when starting another day. One can watch it and show the level to friends. Said another way, TIM teaches the elements of the game, and about games and programming. At the same time, it and teaches and scaffolds strategies for learning in digital spaces. $\underline{38}$ One could not create a puzzle without testing it incessantly, so that learning to test the puzzles teaches a way of thinking and acting conducive to the puzzle design as much as puzzle solving in the free play mode. Most importantly, this skill is transferable. What starts as a lesson about games, in general, becomes a course about the cognitive and affective dimensions of producing digital texts.

The key is that even reaching the free form creative mode, which some would say is the highlight of the game, entails having learned all of the objects, understanding their functionality, and considering their interoperability in order to fully explore the creativity and freedom of the environment. By the third puzzle it becomes apparent that there might be more than one solution or approach for any given puzzle. Thus, any creation needs to anticipate and to plan for such an eventuality. As an aside, when teaching the Programmer's Algorithm with the aid of an online game creator, many students would complain that their finished routines "worked" but they did not receive full marks or, more importantly, affirmation from peers playing them. Negative receptions occur for a number of reasons, particularly in terms of the last three steps of the algorithm. The significance lies in the potential for replay and for sharing the creation with others. Tricks and traps are only interesting the first time. Thus, singular, idiosyncratic, or rigidly narrow solutions have a kind of anti-social function. This, too, provides not only an index of learning, but also of ludicity for a successful game. The importance, as Jensen argues, likes in recognizing the ways that a combination of user-defined goals and rewards that are external to the game can "transform a paidic game into a ludic game." $\underline{39}$ In other words, a seemingly free form game can become rules based through the user's own inputs, manipulations and creations. Again, this entails none of the polarity of Hacker's model, which has the didacticsm of the serious game and the alleged agency of an open environment. Quite the contrary, the open environment can only be exploited once its limits and constraints can be explored and measured. The metanarrative commentary of the 
humor-in the level titles, the functions of the pieces, and the pieces themselves-more than hints at these aspects. The game makes them real.

The need to plan for different sorts of social interaction highlights the importance of testing, debugging and documenting solutions as strategies for learning. That is to say, the social function of a game like TIM becomes more important than the solutions. Games' in-built surveillance in and through social interaction offers a reminder that metacognitive knowledge "consists of both monitoring and control components." 40 This is a cause-effect reversal not encompassed by the determinism of games and/or game scholarship based on the universal presumption of conquest, accumulation and pleasure, nor by the insistence on serious games as the ones that teach. It is no surprise, then, that one finds (pro)social media for Minecraft, Portal, The Stanley Parable, and The Turing Test, for these games (and games like them) instill-indeed, insist on-the same sorts of digital literacies, particularly Minecraft, with its possible worlds, creative mode, and generally scaffolded processes.

Still, TIM differs in its overt playfulness with the very apparatus of learning, both in form and in content. The silliness of the Jack-in-the-box or the spring-loaded boxing glove provide an anti-dote to Minecraft's bland, blocky repetitive rote learning. To put it flatly, Minecraft becomes (nothing but) work at times. $\underline{41}$ While Portal matches silly content and activities, creativity is not as encouraged. The puzzles tend to have one solution, there is no free play, and some of the creativity-like the "high fives" and dancing in co-operative mode-is produced in and through the algorithm of the game; it is neither synthetic nor organic. It is canned. Thus, these games do not comment as overtly on the processes or strategies for learning or offer an index of ludicity. As well, in TIM, any leftover objects do not so much point to extraneous solutions as they provide the impetus and the means for an alternative solution. As well, it might be the case that these are proverbial red herrings or the "MacGuffins," as one might find in a Hitchcock film, but with no proverbial princess to save or points penalty for trying, there is a still an enticement to consider, plan, and implement a solution. Failure then becomes an opportunity to learn. For example, "Puzzle 47: Fetch a Pail" asks that the player deliver two pails from the top of the screen to the bottom of the screen, one on each side. The pails start on treadmills above a series of brick levels. The puzzle can be solved with an elaborate series of explosions using all the dynamite-which can be ignited with a rocket or with a candle-to drop one of the pails, or they can be moved with a series of gears and pulleys using none of the dynamite. In both cases, there are pieces left, which indicates that there might be many, many more solutions. In this way, then, the game provides players with a comment on games as requiring a specific 
set of skills and especially on the determinism of the singular outcome. The player might be better at igniting rockets than igniting candles, better at treadmills and gears than dynamite, or might have a preference for one over another. Whereas some contemporary big-budget releases are hailed for having two or three endings, The Incredible Machine teaches that this can and should be the rule but does not need a didactic approach to do it.

\section{Replay or Advance: Conclusions}

What becomes most clear is that if there is a story that drives The Incredible Machine, it is a metatextual one, simultaneously deterritorializing and extending the realm of possible worlds within, without, and between games. This is a game that teaches: about games, about learning, and about strategies for learning. It does this while suggesting that games are about cooperation, collaboration, and compromise. As well, there is a leveling effect to TIM through its almost complete lack of any need for skill at the traditional game practices. The common requirements for fast-twitches, handeye co-ordination, and quick reflexes are completely eschewed by virtue of The Incredible Machine's pedagogies. It does not matter whether one has fast reflexes or good aim; on the contrary, the game teaches otherwise. Moreover, the game does so without its pedagogies becoming overbearing, didactic, or serious. In fact, these are the hallmarks of so-called "cult" media productions. As Sara Gwenllian-Jones explains in her enumeration of the elements of cult film and television, these types of productions manipulate "[i]ntertextuality, metatextuality, ironic and/or surreal humor, eclecticism, pastiche, and self-referentiality combine to draw viewers." 42 The cognitive and affective response to the textual elements is a sense of belonging to something beyond just the game.

The development of attachments becomes terrifically important given the relationship between cult productions and the "hit-and-run" tactics of resistance Constance Penley attributes to the creators of fan fiction. Taking her cue from de Certeau, Penley observes that such texts enable their creators to "to turn to their own ends forces that systematically exclude or marginalize them." 43 Any puzzle space can become a place that the player, whether as gamer or creator, reconfigures into a space of multiple and simultaneous activities. The arrangement of signs and objects within the game makes TIM a text that defies the deterministic logic of games and of learning with them. The importance of this approach cannot be overstated. In his study of games that strongly prescribe the limits of player performance and manipulation, Michael Skolnik finds that the games actually cause players to "reject the intervention" because they object 
to the overt didacticism, authorial intent, loss of agency, socialization, etc. $\underline{44}$ In contrast, TIM teaches without being explicitly educational so that any player can participate in the cognitive and affective dimensions of learning and enjoyment. As Mayer puts it, games represent and important potential tool for the development of metacognition because they tap people's awareness of "how they process information, their own strengths and weaknesses in processing information, their current level of

proficiency, and the demands of various tasks." 45 Each solution, either for a provided puzzle but especially for a new one, becomes an artifact of the concurrent processes of the in-built and insistent instructional scaffolding.

Moreover, every play-through is a unique opportunity to interpret the space, the components and their limits. Thus, each solution also becomes what de Certeau calls a "practiced place [. . .] a place constituted by a system of signs." $\underline{46}$ Yet each one constitutes something that is different despite its existence in and through the rules of the space. In this way, The Incredible Machine offers a metacognitve pedagogy for how to manipulate systems of logic through the ongoing, active deployment of practices and methods of that system. As de Certeau writes, this kind of play becomes a form of resistance, one that "redistributes its space; it creates at least a certain play in that order, a space for maneuvers of unequal forces and for utopian points of reference." 47 In place of a narrative, The Incredible Machine offers a means of evaluating the products of the very system that produced it. At the same time, it adopts codified systems of teaching and learning to resist the notion that these are somehow apart from digital and/or playful spaces. Indeed, The Incredible Machine shows that strategies for teaching and learning need not be applied rigidly or didactically. Most important, though, it includes as its very kernel a means of developing alternatives to that system. In this last regard, then, The Incredible Machine contributes (not only) insights for considering if and how this process occurs in other games, it also offers its own heuristic, within and without the game, for making more.

\section{Footnotes}

1. The author would like to thank Michelle Ouellette for playing The Incredible Machine with me, as well as offering formative feedback on the paper, and Steven Conway for his kind comments while I was revising. $ヒ$

2. Douglas J. Hacker, "Definitions and Empirical Foundations," in Metacognition in Educational Theory and Practice, eds. Douglas J. Hacker, John Dunlosky, and Arthur 
C. Graesser (New York: Routledge, 1998), 3. $\Perp$

3.

See Christoph Klimmt, Dorothée Hefner, and Peter Vorderer, "The Video Game Experience as 'True' Identification: A Theory of Enjoyable Alterations of Players' SelfPerception," Communication Theory 19, no. 4 (2009): 351-373;

Peter Vorderer, Tilo Hartmann, Christoph Klimmt, "Explaining the Enjoyment of Playing Video Games: The Role of Competition," in ICEC '03: Proceedings of the Second International Conference on Entertainment Computing, Pennsylvania, USA, May 8-10, 2003: 1-9, https://doi.org/10.1145/958720.958735;

Peter Vorderer, Christoph Klimmt, and Ute Ritterfeld, "Enjoyment: At the Heart of Media Entertainment," Communication Theory 14, no. 4 (2004): 388-408, https://doi.org/10.1111/j.1468-2885.2004.tb00321.x..

4. Douglas J. Hacker, "The Role of Metacognition in Learning via Serious Games," in Handbook of Research on Serious Games for Educational Applications, eds. Robert Zheng and Michael K. Gardner (Hershey, PA: IGI Global, 2016), 20.

5. Since it is available online, and it is silly fun, The Incredible Machine is one of the games used in my undergraduate class to demonstrate and practice the stages of the Programmer's Algorithm and different kinds of literacies. $\triangleq$

6. Steven Conway, "Hyper-Ludicity, Contra-Ludicity, and the Digital Game," Eludamos: Journal for Computer Game Culture 4, no. 2 (2010): 135. 7. Graham H. Jensen, "Making Sense of Play in Video Games: Ludus, Paidia, and Possibility Spaces," Eludamos: Journal for Computer Game Culture 7, no. 1 (2013): 72.

8. Rolf F. Nohr, “'Now Let's Continue Testing': Portal and the Rat in the Maze," in "The Cake Is a Lie": Polyperspektivische Betrachtungen Des Computerspiels Am Beispiel von Portal, eds. Britta Neitzel, Thomas Hensel, and Rolf F. Nohr (Münster and Berlin: Lit-Verlag, 2015), 199-224. $\leftrightarrows$

9. Judd Ethan Ruggill and Ken S. McAllister, Gaming Matters: Art, Science, Magic, and the Computer Game Medium (Tuscaloosa, AL: University of Alabama Press, 2011). 
10. Christoph Klimmt, Dorothée Hefner, and Peter Vorderer, "The Video Game Experience as 'True' Identification: A Theory of Enjoyable Alterations of Players' SelfPerception." Communication Theory 19, no. 4 (2009): 351-373; Mary Beth Oliver, et al., "Video Games as Meaningful Entertainment Experiences," Psychology of Popular Media Culture 5, no. 4 (2016): 390-405. $\_$

11. Hacker, "The Role of Metacognition in Learning via Serious Games," 19.

12. Hacker, "The Role of Metacognition in Learning via Serious Games," 20.

13. Hacker, "The Role of Metacognition in Learning via Serious Games," $22 . \sqcup$

14. Here it is important to note that online versions delivered with emulators allow players to access any puzzle at any time. The original game requires that players complete levels to obtain codes that allow access to subsequent ones in the event the player needs or wants to continue in another session.

15. Espen Aarseth, "Genre Trouble: Narrativism and the Art of Simulation," in First Person: New Media as Story, Performance, and Game, eds. Noah Wardrip-Fruin and Pat Harrigan (Cambridge, MA: MIT Press, 2004), 51.

16. Alexander Galloway, Gaming: Essays on Algorithmic Culture (Minneapolis: University of Minnesota Press, 2006). $\doteq$

17. Marc Ouellette and Michelle Ouellette, "Make Lemonade: The Pleasantly Unpleasant Aesthetics of Playing Portal," in "The Cake Is a Lie": Polyperspektivische Betrachtungen Des Computerspiels Am Beispiel von Portal, eds. Britta Neitzel, Thomas Hensel, and Rolf F. Nohr (Münster and Berlin: Lit-Verlag, 2015), 151-173. 18. Bernard Perron, "From Gamers to Players and Gameplayers: The Example of Interactive Movies," in The Video Game Theory Reader, eds. Mark J. P. Wolf and Bernard Perron (New York: Routledge, 2004), 242.

19. Hacker, "The Role of Metacognition in Learning via Serious Games," $25 . \Leftarrow$ 20.

Michel de Certeau, The Practice of Everyday Life (Berkeley, CA: University of California Press, 1988), 18.

Indeed, as is cited, in Gaming Matters, Ruggill and McAllister open with an extended discussion of the widening gap between the two camps in game studies, 
based on the distinction between what games are and what they can do. As I argue, $T I M$ provides linkages. $\_$

21. Rolf F. Nohr, “'Now Let's Continue Testing.'”

22. Jensen, "Making Sense of Play in Video Games."

23.

Bradley J. Fest, "Metaproceduralism: The Stanley Parable and the Legacies of Postmodern Metafiction," Wide Screen 6, no. 1 (2016): 1-23.

Beyond The Stanley Parable and the previously mentioned Portal series, The Turing Test (which is a Portal knockoff) could be added to the list of similar games involving puzzles, metagaming features, and the need to develop an algorithm. As well, The Witness requires the player to solve a set of puzzles to escape an island. The game has no instructions and so learning from each puzzle is necessary to solve the next. The final solution, the mystery of how you got there, is itself a metagame element. That said, competitive and violent games like the immensely popular Defense Grid combines these same elements. However, it is also worth noting that an underexamined feature of this game is its debt to the classic Pipe Dreams, which requires players to quickly place water pipes on a grid in order to avoid a break in the flow. These games also require learning the elements and their combinations, and the ludicity of the games allows for multiple and simultaneous solutions as well as facilitating strategies for learning. These games will be mentioned elsewhere. $\bullet$ 24. Judd Ethan Ruggill, Ken S. McAllister, and David Menchaca, "The Gamework," Communication and Critical/Cultural Studies 1, no. 4 (2004): 297-312.

25. Ruggill et al., "The Gamework,” 301.

26.

Michelle E. Ouellette and Marc A. Ouellette, "Married, with Children and an XBox: Compromise in Video Game Play," in Everyday Play, ed. Samuel Tobin, The New Everyday: A Media Commons Project (2013), http://mediacommons.org/tne/pieces/married-children-and-xbox-compromise-videogame-play.

While the obvious comparison is to the puzzle-based Portal 2, these analyses also extend to show the ways other games in other genres, including Modern Warfare, Grand Theft Auto, and others can be played co-operatively, even in the first-person 
mode. The practice of alternating the levels with a partner constitutes a metagame practice and necessitates comprises for it requires learning and adapting to the style and habits of another gamer. $ヒ$

27. Hacker, "The Role of Metacognition in Learning via Serious Games," 21. 28. Richard E. Mayer, "The Role of Metacognition in STEM Games and Simulations," in Using Games and Simulations for Teaching and Assessment: Key Issues, eds. Harold F. O’Neil, Eva L. Baker, and Ray S. Perez (New York: Routledge, 2016), 190.

29. Mayer, "The Role of Metacognition in STEM Games and Simulations," 22.

30. Aarseth, "Genre Trouble," 51.

31. Perron, "From Gamers to Players and Gameplayers," 249.

32. Perron, "From Gamers to Players and Gameplayers," 252.

33. It could be argued that this is a method and not an algorithm because there are no calculations involved. However, the process does require and encompass an iterative process. $\triangleq$

34. Hacker, "The Role of Metacognition in Learning via Serious Games," 21. 35. Mayer, "The Role of Metacognition in STEM Games and Simulations," 183. 36. Hacker, "Definitions and Empirical Foundations," 3.

37. Hacker, "The Role of Metacognition in Learning via Serious Games," 22. 38. Indeed, there are YouTube videos for solutions to the original puzzles and, in keeping with the rise of "let's play," videos of user-developed content. 39. Jensen, "Making Sense of Play in Video Games," 73. $\subseteq$ 40. Hacker, "The Role of Metacognition in Learning via Serious Games," 21. 41. Helping my children mine for diamonds reminds me that games belong to a governmental system, one which prepares them for lives as worker/consumers as opposed to citizens. $\leftrightarrows$ 
42. Sara Gwenllian-Jones, "Virtual Reality and Cult Television," in Cult Television, eds. Sara Gwenllian-Jones and Roberta E. Pearson (Minneapolis, MN: University of Minnesota Press, 2004), xv. $\_$

43. Constance Penley, "Brownian Motion: Women, Tactics, and Technology," in Technoculture, eds. Constance Penley and Andrew Ross (Minneapolis, MN: University of Minnesota Press, 1991), 139.

44. Michael Skolnik, "Strong and Weak Procedurality," Journal of Gaming \& Virtual Worlds 5, no. 2 (2013): 157.

45. Mayer, "The Role of Metacognition in STEM Games and Simulations," 183. $\_$ 46. de Certeau, The Practice of Everyday Life, 117. 47. de Certeau, The Practice of Everyday Life, 18. 